\section{Biocatalytic Directed Cationic Cyclization Cascades toward Fragrance Ingredients}

\section{Category}

Organo- and Biocatalysis

Key words

squalene-hopene cyclase

directed cyclization cascade reaction molecular anchoring monocyclization

fragrances
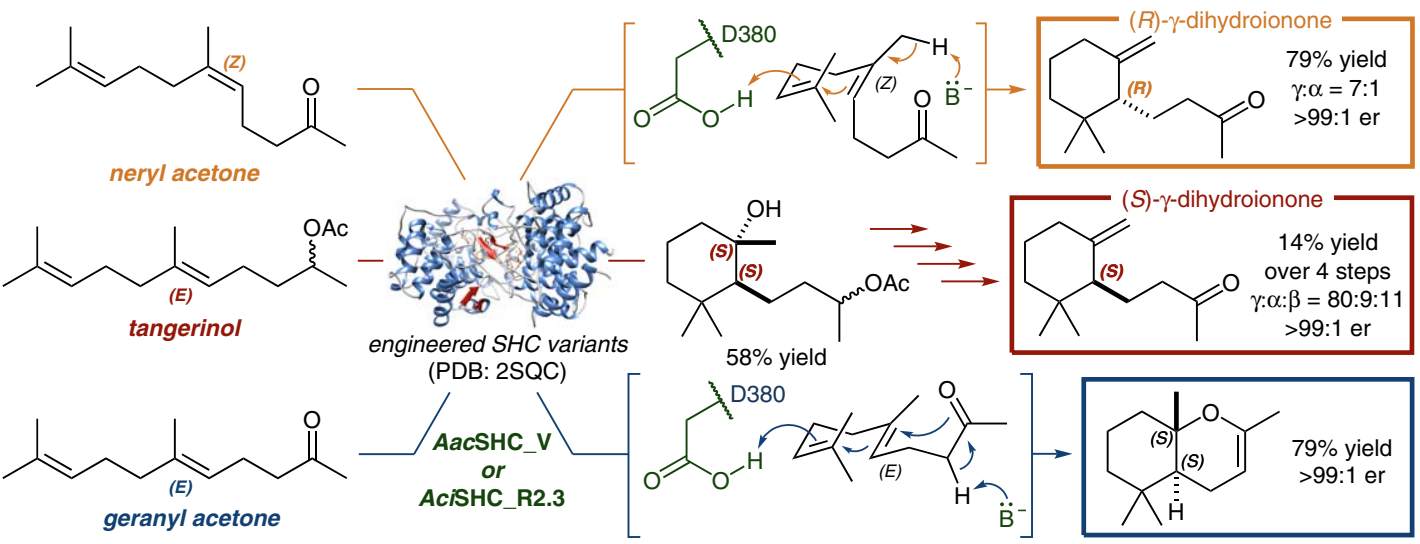

- Directed cascade approach / proposed mechanism for the stereoselective monocyclization

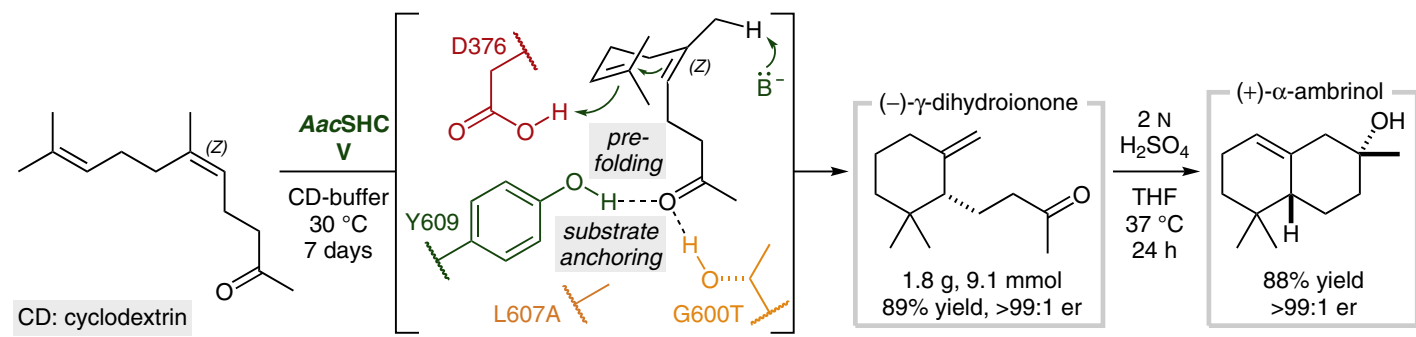

Significance: The Hauer group and a collaborative effort between the groups of Buller and Bornscheuer and Givaudan independently report the development of engineered squalene-hopene cyclases (SHCs) for stereoselective, cationic cyclization cascades toward natural products and, in particular, fragrance ingredients. The Hauer group describes a biocatalytic monocyclization of neryl acetone by using an engineered SHC from Alicyclobacillus acidocaldarius (AacSHC) to give $(R)$ - $\gamma$-dihydroionone in excellent yield and enantioselectivity. Buller and collaborators report a complimentary stereodivergent approach from either neryl or geranyl acetone by using variants based on a previously unidentified SHC from Acidothermus cellulolyticus (AciSHC).
Comment: Cationic cyclization cascades serve as powerful tools for the rapid assembly of complex carbon skeletons in a highly efficient manner. However, stereoselective methods to interrupt the cascade at a desired intermediate to preferentially access monocyclic products are challenging and underdeveloped. The Hauer group devised a strategy to overcome this limitation by engineering the active site of an SHC. Specific addition of stabilizing hydrogen-bonding interactions for substrate anchoring favors deprotonation over the usual bicycle formation. Buller and collaborators demonstrated that the stereoselectivity of the cyclization is governed by the double-bond geometry of the starting material, due to identical prefolding of the chair. 